\title{
LLEUQUE-INIA, NEW HIGH YIELD SPRING DURUM WHEAT VARIETY FOR CHILE
}

\author{
Iván Matus $^{1 *}$, Ricardo Madariaga1, Christian Alfaro², and Claudio Jobet ${ }^{3}$
}

\begin{abstract}
The Lleuque-INIA spring durum wheat variety (Triticum turgidum var. durum L.) originated from a cross carried out by the Instituto de Investigaciones Agropecuarias (INIA), National Wheat Program, in the Centro Regional de Investigación La Platina, Santiago, Chile, in 1993. This variety has an upright growth habit in the seedling stage; the adult plant is of medium height and varies between 80 and $90 \mathrm{~cm}$. The spike is medium-sized, white, and exhibits long whitish awns along its full length. The grain is large-sized, elongated, golden yellow, and vitreous. The variety was sown in mid-August in the Santa Rosa Experimental Station ( $36^{\circ} 31^{\prime}$ S; $71^{\circ} 54^{\prime}$ W), Chillán, head emergence occurred between 89 and $91 \mathrm{~d}$ after sowing, and was 6 to $8 \mathrm{~d}$ later than 'Llareta-INIA' and 'Corcolén-INIA'. In La Platina, 'Lleuque-INIA' had a mean yield of 16.7 and $20.8 \%$ higher than vars. Llareta-INIA and Corcolén-INIA, respectively, whereas the yield was higher in Chillán by 10.3 and $10.1 \%$, respectively. On the other hand, in Yungay located in the Ñuble foothills, 'Lleuque-INIA' yield exceeded 'Llareta-INIA' by $13.07 \%$ and var. Corcolén-INIA by $16.97 \%$. In Humán, var. Lleuque-INIA yield exceeded the control vars. Llareta-INIA and Corcolén-INIA by 15.23 and $24.03 \%$, respectively. Lleuque-INIA variety is a type of wheat with good hectoliter weight, good wet gluten content (\%), and a protein value mean fluctuating between 10.47 and $12.3 \%$.
\end{abstract}

Key words: Durum wheat, Triticum turgidum var. durum, Lleuque-INIA.

$\mathrm{D}$ urum wheat (Triticum turgidum var. durum L.) in Chile is destined to the manufacture of noodles and pasta. It has large-sized grains, a vitreous aspect, hard texture, high hectoliter weight, and yellow pigment. This cereal has been cultivated in Chile since the 1950s and represents about $10 \%$ of the area sown with bread wheat. The traditional cultivation area has been moved from the central-northern zone of the country to the centralsouthern zone. 'Lleuque-INIA' is the first released product variety of the selection carried out in the central-southern zone of the country.

\section{Origin}

'Lleuque-INIA' is a spring durum wheat variety originated from a cross carried out in 1993 by the National Wheat Program of Instituto de Investigaciones Agropecuarias

\footnotetext{
${ }^{1}$ Instituto de Investigaciones Agropecuarias INIA, Casilla 426, Chillán, Chile. "Corresponding author (imatus@inia.cl).

${ }^{2}$ Instituto de Investigaciones Agropecuarias INIA, Casilla 13, Rengo, Chile.

${ }^{3}$ Instituto de Investigaciones Agropecuarias INIA, Casilla 58-D, Temuco, Chile.

Received: 29 September 2010.

Accepted: 4 January 2011.
}

(INIA) in the Centro Regional de Investigación (Regional Research Center) (CRI) La Platina (333' S; 70³8' W), Santiago, Chile. A mass selection was carried out in 1995 on the $F_{2}$ generation. The $F_{3}$ to $F_{5}$ selection stages were carried out between 1996 and 1998 using the pedigree method. A preliminary yield trial was evaluated in 1999 and included in the main yield trial in the year 2000 . Regional bread wheat trials of CRI La Platina were studied in 2001 and 2002. Standard bread wheat trials of CRI-Quilamapu (36 $31^{\prime}$ S; 71 $54^{\circ}$ ' W) were studied between 2004 and 2008. These trials were registered in 2005 and 2006 as variety trials applying for Registration of Varieties Suitable for Certification (RVSC), condition required by the Ministry of Agriculture through the Servicio Agrícola y Ganadero (Agriculture and Livestock Service). The experimental line for all these trials was identified as V-116.

\section{Crossing and pedigree}

The following is the genealogy of Lleuque-INIA: YEL'S'/BAR'S'/3/GR'S'/AFN//CR'S'/5/DOM'S'// CR'S'*2/GS'S'/3/SCO'S'/4/HORA/6/LAP76/ GULL'S'/7/LICAN

A-27735-0P-3P-1P-1P 


\section{Morphological description of the plant}

This durum wheat habitually develops in the spring and has an upright growth habit in the seedling stage (Figure 1). The adult plant is of medium height and varies between 80 and $90 \mathrm{~cm}$ (Figure 2). Glaucocity of the flag leaf sheath is medium. The leaf blade (underside) of the flag leaf exhibits nil or weak glaucocity. Glaucocity is medium in the spike neck during anthesis. Stalk medulla is medium and has low to moderate resistance to lodging.

\section{Spike and grain characteristics}

The spike is medium-sized, compact, white, and with high glaucocity in anthesis, has parallel edges, and exhibits long whitish awns along its full length (Figure 3). The peak of the lower glume is very long and the bottom does not exhibit hairiness.

The grain is large-sized, elongated, golden yellow, and vitreous. The weight of 1000 seeds is 65 to $70 \mathrm{~g}$.

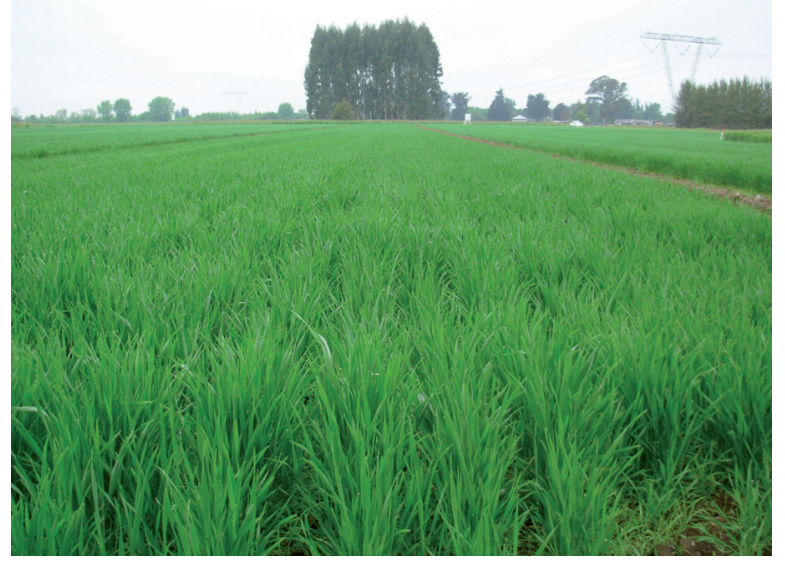

Figure 1. Lleuque-INIA planted in Chillan. Santa Rosa Experimental Station.

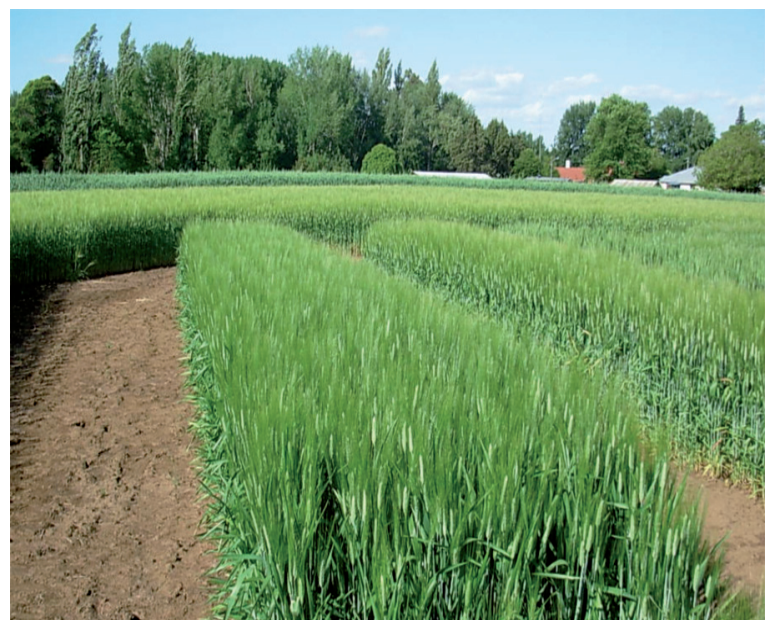

Figure 2. Adult plant of Lleuque-INIA planted in Yungay, a location of the foothills of Nuble province.

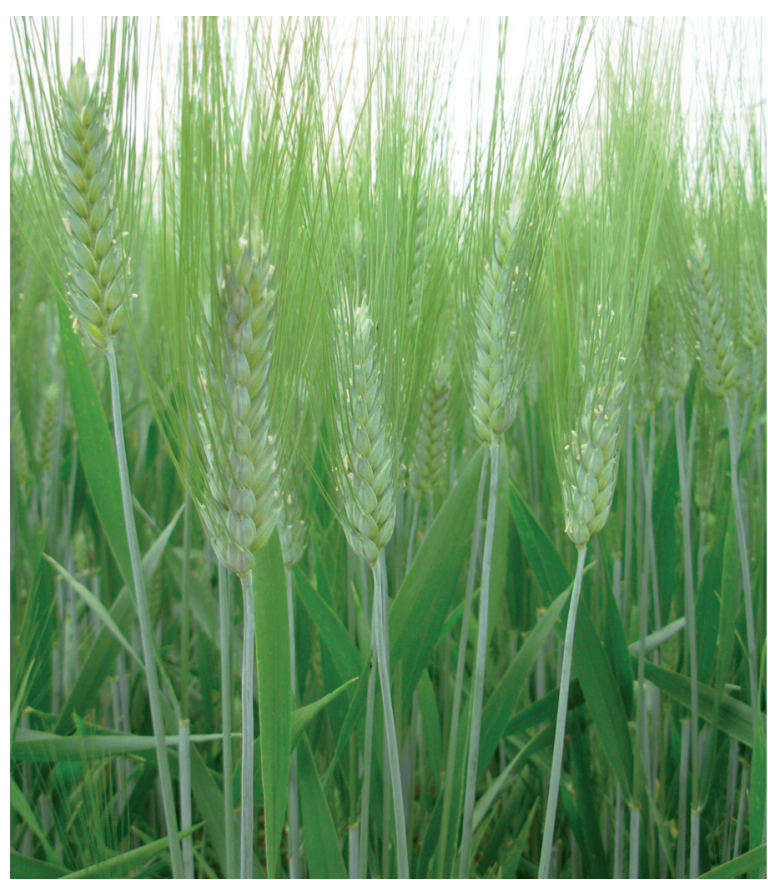

Figure 3. Spike of Lleuque-INIA at anthesis.

\section{Agronomic and phytopathological characteristics}

The variety was sown in mid-August in the Santa Rosa Experimental Station ( $36^{\circ} 31^{\prime}$ S; $71^{\circ} 54^{\prime}$ W), Chillán, head emergence occurred between 89 and $91 \mathrm{~d}$ after sowing, and was 6 to $8 \mathrm{~d}$ later than 'Llareta-INIA' (Ramírez et al., 1997) and 'Corcolén-INIA'.

Up to the 2008-2009 season, 'Lleuque-INIA' showed resistance to stripe rust (Puccinia striiformis West. f. sp. tritici), leaf rust (Puccinia triticina Erikss.), and resistance to moderate resistance to powdery mildew caused by the Blumeria graminis DC. f. sp. tritici Marchal fungus (Tables 1 and 2).

\section{Grain yield}

From 2004 to 2008, 'Lleuque-INIA' was evaluated in standard trials conducted in irrigated soils in the provinces of Santiago (CRI La Platina, 333' S; 70³8' W), Nuble (Santa Rosa Experimental Station, $36^{\circ} 31^{\prime}$ S; $71^{\circ} 54^{\prime}$ W), and Yungay (Ñuble foothills $37^{\circ} 08^{\prime} \mathrm{S} ; 72^{\circ} 0^{\prime} \mathrm{W}$ ), and Bío Bío (Humán Experimental Center in Los Ángeles $\left.37^{\circ} 26^{\prime} \mathrm{S} ; 7^{\circ} 14^{\prime} \mathrm{W}\right)$. 'Lleuque-INIA' had a mean yield of 16.7 and $20.8 \%$ higher than the vars. Llareta-INIA and Corcolén-INIA, respectively, in La Platina. Yield in Chillán was 10.3 and $10.1 \%$ higher than 'Llareta-INIA' and 'Corcolén-INIA', respectively. On the other hand, 'Lleuque-INIA' yield in Yungay was higher than 'LlaretaINIA' by $13.07 \%$ and 'Corcolén-INIA' by $16.97 \%$. 'Lleuque-INIA' yield in Humán was also higher than the control vars. Llareta-INIA and Corcolén-INIA by 15.23 and $24.03 \%$, respectively (Table 3 ). 
Table 1. Behavior of stripe rust (Puccinia striiformis), leaf rust (P. triticina), and powdery mildew (Blumeria graminis) of cv. Lleuque-INIA compared to control cv. Llareta-INIA in four irrigated locations and five agricultural cycles.

\begin{tabular}{|c|c|c|c|c|c|c|c|}
\hline \multirow[b]{2}{*}{ Location } & \multirow[b]{2}{*}{ Year } & \multicolumn{3}{|c|}{ Lleuque-INIA } & \multicolumn{3}{|c|}{ Llareta-INIA } \\
\hline & & $\begin{array}{l}\text { Yellow } \\
\text { rust }^{1}\end{array}$ & $\begin{array}{l}\text { Leaf } \\
\text { rust }^{1}\end{array}$ & $\begin{array}{c}\text { Powdery } \\
\text { mildew }\end{array}$ & $\begin{array}{c}\text { Yellow } \\
\text { rust }^{1}\end{array}$ & $\begin{array}{l}\text { Leaf } \\
\text { rust }^{1}\end{array}$ & $\begin{array}{l}\text { Powdery } \\
\text { mildew }\end{array}$ \\
\hline \multirow[t]{5}{*}{ La Platina } & 2004 & 0 & 0 & 2 & 0 & 0 & 3 \\
\hline & 2005 & 0 & 0 & 0 & 0 & 0 & 0 \\
\hline & 2006 & 0 & 0 & 0 & 0 & 0 & 0 \\
\hline & 2007 & 0 & 0 & 0 & 0 & 0 & 0 \\
\hline & 2008 & 0 & 0 & 0 & 0 & 0 & 0 \\
\hline \multirow[t]{5}{*}{ Chillán } & 2004 & 0 & 0 & 0 & 0 & 0 & 7 \\
\hline & 2005 & TMR & 0 & 0 & TMR & 0 & 0 \\
\hline & 2006 & 0 & 0 & 0 & TMR & 0 & 0 \\
\hline & 2007 & 0 & 0 & 0 & 0 & 0 & 0 \\
\hline & 2008 & 0 & 0 & 0 & 0 & 0 & 0 \\
\hline \multirow[t]{5}{*}{ Yungay } & 2004 & 0 & 0 & 4 & 0 & 0 & 8 \\
\hline & 2005 & 0 & 0 & 0 & 0 & 0 & 0 \\
\hline & 2006 & 0 & 0 & 0 & 0 & 0 & 0 \\
\hline & 2007 & 0 & 0 & 0 & 0 & 0 & 0 \\
\hline & 2008 & 0 & 0 & 0 & 0 & 0 & 0 \\
\hline \multirow[t]{5}{*}{ Humán } & 2004 & 0 & 0 & 2 & 0 & 0 & 6 \\
\hline & 2005 & 0 & 0 & 0 & 0 & 0 & 0 \\
\hline & 2006 & 0 & 0 & 0 & $5 \mathrm{MS}$ & 0 & 0 \\
\hline & 2007 & $5 \mathrm{MR}$ & 0 & 0 & TMS & 0 & 0 \\
\hline & 2008 & 0 & 0 & 0 & 0 & 0 & 0 \\
\hline
\end{tabular}

${ }^{1}$ Values according to modified Cobb Scale (Peterson et al., 1984) where attack intensity can vary between 0 and $100 \%(\mathrm{~T}=$ traces), plant reaction can be: resistant (R), moderately resistant (MR), moderately susceptible (MS), or susceptible (S).

${ }^{2}$ Values Saari and Prescott (1975) scale. Scale from 1 to 9.

Table 2. Behavior of stripe rust (Puccinia striiformis), leaf rust (P. triticina), and powdery mildew (Blumeria graminis) of cv. Lleuque-INIA compared to control cv. Corcolén-INIA in four irrigated locations and five agricultural cycles.

\begin{tabular}{|c|c|c|c|c|c|c|c|}
\hline \multirow[b]{2}{*}{ Location } & \multirow[b]{2}{*}{ Year } & \multicolumn{3}{|c|}{ Lleuque-INIA } & \multicolumn{3}{|c|}{ Llareta-INIA } \\
\hline & & $\begin{array}{c}\text { Yellow } \\
\text { rust }^{1}\end{array}$ & $\begin{array}{l}\text { Leaf } \\
\text { rust }^{1}\end{array}$ & $\begin{array}{l}\text { Powdery } \\
\text { mildew }^{2}\end{array}$ & $\begin{array}{l}\text { Yellow } \\
\text { rust }^{1}\end{array}$ & $\begin{array}{l}\text { Leaf } \\
\text { rust }^{1}\end{array}$ & $\begin{array}{l}\text { Powdery } \\
\text { mildew }\end{array}$ \\
\hline \multirow[t]{5}{*}{ La Platina } & 2004 & 0 & 0 & 2 & 0 & 0 & 2 \\
\hline & 2005 & 0 & 0 & 0 & 0 & 0 & 0 \\
\hline & 2006 & 0 & 0 & 0 & 0 & 0 & 0 \\
\hline & 2007 & 0 & 0 & 0 & 0 & 0 & 0 \\
\hline & 2008 & 0 & 0 & 0 & 0 & 0 & 0 \\
\hline \multirow[t]{5}{*}{ Chillán } & 2004 & 0 & 0 & 0 & 0 & 0 & 4 \\
\hline & 2005 & TMR & 0 & 0 & TMR & 0 & 0 \\
\hline & 2006 & 0 & 0 & 0 & TMS & 0 & 0 \\
\hline & 2007 & 0 & 0 & 0 & $5 \mathrm{MS}$ & 0 & 0 \\
\hline & 2008 & 0 & 0 & 0 & 0 & 0 & 0 \\
\hline \multirow[t]{5}{*}{ Yungay } & 2004 & 0 & 0 & 4 & 0 & 0 & 6 \\
\hline & 2005 & 0 & 0 & 0 & 0 & 0 & 0 \\
\hline & 2006 & 0 & 0 & 0 & 0 & 0 & 0 \\
\hline & 2007 & 0 & 0 & 0 & 0 & 0 & 0 \\
\hline & 2008 & 0 & 0 & 0 & 0 & 0 & 0 \\
\hline \multirow{5}{*}{ Humán } & 2004 & 0 & 0 & 2 & 0 & 0 & 4 \\
\hline & 2005 & 0 & 0 & 0 & $5 \mathrm{MS}$ & 0 & 0 \\
\hline & 2006 & 0 & 0 & 0 & $10 \mathrm{MS}$ & 0 & 0 \\
\hline & 2007 & $5 \mathrm{MR}$ & 0 & 0 & TMS & 0 & 0 \\
\hline & 2008 & 0 & 0 & 0 & 0 & 0 & 0 \\
\hline
\end{tabular}

${ }^{1}$ Values according to modified Cobb Scale (Peterson et al., 1984) where attack intensity can vary between 0 and $100 \%(\mathrm{~T}=$ traces), plant reaction can be: resistant (R), moderately resistant (MR), moderately susceptible (MS), or susceptible (S).

${ }^{2}$ Values Saari and Prescott Scale (1975). Scale from 1 to 9. 
Table 3. Grain yield of cv. Lleuque-INIA compared to control cvs. Llareta-INIA and Corcolén-INIA standard trials conducted in four irrigated locations from 2004 to 2008 .

\begin{tabular}{|c|c|c|c|c|}
\hline \multirow[b]{2}{*}{ Location } & \multirow[b]{2}{*}{ Year } & \multicolumn{3}{|c|}{ Cultivars } \\
\hline & & $\begin{array}{l}\text { Lleuque- } \\
\text { INIA }\end{array}$ & $\begin{array}{l}\text { Llareta- } \\
\text { INIA }\end{array}$ & $\begin{array}{l}\text { Corcolén- } \\
\text { INIA }\end{array}$ \\
\hline & & & $-\mathrm{tha}^{-1}$ & \\
\hline \multirow[t]{6}{*}{ La Platina } & 2004 & $5.31 \mathrm{a}$ & $4.93 b$ & $3.84 \mathrm{c}$ \\
\hline & 2005 & $6.55 \mathrm{a}$ & $4.91 \mathrm{~b}$ & $5.09 \mathrm{~b}$ \\
\hline & 2006 & $4.33 \mathrm{a}$ & $4.12 \mathrm{a}$ & $4.34 \mathrm{a}$ \\
\hline & 2007 & $9.62 \mathrm{a}$ & $10.14 a$ & $9.33 \mathrm{a}$ \\
\hline & 2008 & $7.49 \mathrm{a}$ & $4.44 b$ & $4.95 b$ \\
\hline & Mean & 6.66 & 5.71 & 5.51 \\
\hline \multirow[t]{6}{*}{ Chillán } & 2004 & $12.01 \mathrm{a}$ & $8.84 \mathrm{c}$ & $10.23 b$ \\
\hline & 2005 & $9.98 \mathrm{a}$ & $10.22 \mathrm{a}$ & $8.74 b$ \\
\hline & 2006 & $7.17 \mathrm{a}$ & $6.33 b$ & $6.91 \mathrm{a}$ \\
\hline & 2007 & $10.51 \mathrm{a}$ & $9.72 \mathrm{a}$ & $8.71 b$ \\
\hline & 2008 & $9.29 \mathrm{a}$ & $9.25 \mathrm{a}$ & $9.87 \mathrm{a}$ \\
\hline & Mean & 9.79 & 8.87 & 8.89 \\
\hline \multirow[t]{6}{*}{ Yungay } & 2004 & $8.14 a$ & $6.36 \mathrm{~b}$ & $7.70 \mathrm{a}$ \\
\hline & 2005 & $8.34 b$ & $9.78 \mathrm{a}$ & $8.42 b$ \\
\hline & 2006 & $10.89 \mathrm{a}$ & $8.72 b$ & $9.09 \mathrm{~b}$ \\
\hline & 2007 & $8.16 \mathrm{a}$ & $7.49 \mathrm{a}$ & $6.06 \mathrm{~b}$ \\
\hline & 2008 & $9.12 \mathrm{a}$ & $7.14 b$ & $6.89 b$ \\
\hline & Mean & 8.93 & 7.89 & 7.63 \\
\hline \multirow[t]{6}{*}{ Humán } & 2004 & $12.25 \mathrm{a}$ & $9.29 \mathrm{a}$ & $9.89 a$ \\
\hline & 2005 & $12.36 \mathrm{a}$ & $11.59 \mathrm{a}$ & $9.68 \mathrm{~b}$ \\
\hline & 2006 & $11.65 \mathrm{a}$ & $10.68 \mathrm{a}$ & $10.80 \mathrm{a}$ \\
\hline & 2007 & $9.81 \mathrm{a}$ & $9.35 \mathrm{a}$ & $7.77 \mathrm{~b}$ \\
\hline & 2008 & $8.51 \mathrm{a}$ & $6.44 b$ & $5.85 \mathrm{~b}$ \\
\hline & Mean & 10.92 & 9.47 & 8.79 \\
\hline
\end{tabular}

Different letters between cultivars for each year and location are statistically different at $\mathrm{P}<0.01$.

\section{Quality}

Lleuque-INIA is a variety of wheat with mean hectoliter weight between 81 and $84 \mathrm{~kg} \mathrm{hL}^{-1}$, wet gluten mean percentages between 28.83 and $35.66 \%$, and mean protein values fluctuating between 10.47 and $12.3 \%$ (Tables 4, 5, and 6). In La Platina, hectoliter weight was low for the three varieties in 2004 because of viruses (Table 4). The grain showed a very low yellow berry percentage (Table 7) and very low incidence of black tip (Table 8). Low levels of black tip over time in the different locations must also be highlighted.
Table 4. Hectoliter weight of cv. Lleuque-INIA compared to control cvs. Llareta-INIA and Corcolén-INIA standard trials conducted in four irrigated locations from 2004 to 2008.

\begin{tabular}{|c|c|c|c|c|}
\hline \multirow[b]{2}{*}{ Location } & \multirow[b]{2}{*}{ Year } & \multicolumn{3}{|c|}{ Cultivars } \\
\hline & & $\begin{array}{l}\text { Lleuque- } \\
\text { INIA }\end{array}$ & $\begin{array}{l}\text { Llareta- } \\
\text { INIA }\end{array}$ & $\begin{array}{l}\text { Corcolén- } \\
\text { INIA }\end{array}$ \\
\hline & & & $-\mathrm{kg} \mathrm{hL}^{-1}$ & \\
\hline \multirow[t]{6}{*}{ La Platina } & 2004 & 77.60 & 79.90 & 79.20 \\
\hline & 2005 & 84.15 & 84.38 & 84.98 \\
\hline & 2006 & 81.04 & 82.12 & 82.31 \\
\hline & 2007 & 83.68 & 85.52 & 85.30 \\
\hline & 2008 & 82.36 & 82.44 & 83.22 \\
\hline & Mean & 81.77 & 82.87 & 83.00 \\
\hline \multirow[t]{6}{*}{ Chillán } & 2004 & 85.01 & 85.61 & 86.46 \\
\hline & 2005 & 82.33 & 83.88 & 83.75 \\
\hline & 2006 & 83.90 & 85.42 & 85.68 \\
\hline & 2007 & 85.46 & 86.86 & 86.28 \\
\hline & 2008 & 87.67 & 88.12 & 87.83 \\
\hline & Mean & 84.87 & 85.98 & 86.00 \\
\hline \multirow[t]{6}{*}{ Yungay } & 2004 & 79.59 & 80.25 & 81.55 \\
\hline & 2005 & 83.52 & 83.89 & 83.86 \\
\hline & 2006 & 84.64 & 85.04 & 85.77 \\
\hline & 2007 & 82.98 & 84.64 & 83.96 \\
\hline & 2008 & 83.99 & 84.75 & 84.85 \\
\hline & Mean & 82.94 & 83.71 & 83.99 \\
\hline \multirow[t]{6}{*}{ Humán } & 2004 & 82.76 & 82.48 & 83.48 \\
\hline & 2005 & 84.50 & 85.32 & 84.82 \\
\hline & 2006 & 84.32 & 85.22 & 85.69 \\
\hline & 2007 & 87.31 & 87.20 & 86.81 \\
\hline & 2008 & 85.11 & 86.70 & 86.22 \\
\hline & Mean & 84.80 & 85.38 & 85.40 \\
\hline
\end{tabular}

Mean values of four replicates.

\section{Cultivation area and sowing dates}

Data obtained in the regional and standard trials allow recommending 'Lleuque-INIA' for the zone between the Metropolitan Region to the Bío Bío Region.

Sowing in irrigated soils is recommended between June and August. This variety can be sown earlier than the vars. Llareta-INIA and Corcolén-INIA. 
Table 5. Wet gluten of cv. Lleuque-INIA compared to control cvs. Llareta-INIA and Corcolén-INIA standard trials conducted in four irrigated locations from 2004 to 2008 .

\begin{tabular}{|c|c|c|c|c|}
\hline \multirow[b]{2}{*}{ Location } & \multirow[b]{2}{*}{ Year } & \multicolumn{3}{|c|}{ Cultivars } \\
\hline & & $\begin{array}{l}\text { Lleuque- } \\
\text { INIA }\end{array}$ & $\begin{array}{l}\text { Llareta- } \\
\text { INIA }\end{array}$ & $\begin{array}{l}\text { Corcolén- } \\
\text { INIA }\end{array}$ \\
\hline & & & $-\%$ & \\
\hline \multirow[t]{6}{*}{ La Platina } & 2004 & 34.14 & 37.17 & 35.33 \\
\hline & 2005 & 23.49 & 35.36 & 25.96 \\
\hline & 2006 & 31.72 & 32.52 & 29.54 \\
\hline & 2007 & 26.30 & 28.00 & 22.70 \\
\hline & 2008 & 28.50 & 29.40 & 25.90 \\
\hline & Mean & 28.83 & 32.49 & 27.89 \\
\hline \multirow[t]{6}{*}{ Chillán } & 2004 & 40.29 & 42.31 & 38.00 \\
\hline & 2005 & 25.63 & 38.97 & 33.99 \\
\hline & 2006 & 31.67 & 35.45 & 32.83 \\
\hline & 2007 & 26.80 & 33.40 & 38.20 \\
\hline & 2008 & 20.40 & 27.85 & 23.85 \\
\hline & Mean & 28.96 & 35.59 & 33.37 \\
\hline \multirow[t]{6}{*}{ Yungay } & 2004 & 32.06 & 30.88 & 28.99 \\
\hline & 2005 & 46.68 & 49.25 & 49.43 \\
\hline & 2006 & 33.26 & 38.22 & 34.30 \\
\hline & 2007 & 34.90 & 35.10 & 42.60 \\
\hline & 2008 & 28.90 & 40.15 & 34.90 \\
\hline & Mean & 35.16 & 38.72 & 38.04 \\
\hline \multirow[t]{6}{*}{ Humán } & 2004 & 37.60 & 34.53 & 36.20 \\
\hline & 2005 & 44.78 & 39.61 & 44.46 \\
\hline & 2006 & 34.45 & 42.81 & 40.05 \\
\hline & 2007 & 38.00 & 40.80 & 39.00 \\
\hline & 2008 & 23.45 & 29.40 & 29.50 \\
\hline & Mean & 35.66 & 39.44 & 37.84 \\
\hline
\end{tabular}

Wet gluten (\%): < 20 low, 21-25 normal, 26-30 good, > 30 very good. Mean values of two replicates.
Table 6. Protein of cv. Lleuque-INIA compared to control cvs. Llareta-INIA and Corcolén-INIA standard trials conducted in four irrigated locations from 2004 to 2008.

\begin{tabular}{|c|c|c|c|c|}
\hline \multirow[b]{2}{*}{ Location } & \multirow[b]{2}{*}{ Year } & \multicolumn{3}{|c|}{ Cultivars } \\
\hline & & $\begin{array}{l}\text { Lleuque- } \\
\text { INIA }\end{array}$ & $\begin{array}{l}\text { Llareta- } \\
\text { INIA }\end{array}$ & $\begin{array}{c}\text { Corcolén- } \\
\text { INIA }\end{array}$ \\
\hline & & & $-\%$ & 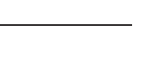 \\
\hline \multirow[t]{4}{*}{ La Platina } & 2006 & 12.60 & 12.60 & 12.10 \\
\hline & 2007 & 11.10 & 11.20 & 10.75 \\
\hline & 2008 & 12.80 & 13.60 & 12.50 \\
\hline & Mean & 12.17 & 12.47 & 11.78 \\
\hline \multirow[t]{4}{*}{ Chillán } & 2006 & 11.60 & 11.90 & 11.70 \\
\hline & 2007 & 10.10 & 10.90 & 11.80 \\
\hline & 2008 & 9.70 & 11.00 & 10.80 \\
\hline & Mean & 10.47 & 11.27 & 11.43 \\
\hline \multirow[t]{4}{*}{ Yungay } & 2006 & 11.55 & 12.10 & 12.05 \\
\hline & 2007 & 11.80 & 11.65 & 12.60 \\
\hline & 2008 & 11.30 & 12.80 & 12.40 \\
\hline & Mean & 11.55 & 12.18 & 12.35 \\
\hline \multirow[t]{4}{*}{ Humán } & 2006 & 12.65 & 13.30 & 12.85 \\
\hline & 2007 & 12.45 & 13.25 & 13.75 \\
\hline & 2008 & 11.80 & 12.10 & 11.20 \\
\hline & Mean & 12.30 & 12.88 & 12.60 \\
\hline
\end{tabular}

Protein (\%): < 8 low; 8.1 to 9.5 normal; 9.6 to 11 good.

Mean values of two replicates. 
Table 7. Yellow berry (\%) of cv. Lleuque-INIA compared to control cvs. Llareta-INIA and Corcolén-INIA standard trials conducted in four irrigated locations from 2004 to 2008 .

\begin{tabular}{|c|c|c|c|c|}
\hline \multirow[b]{2}{*}{ Location } & \multirow[b]{2}{*}{ Year } & \multicolumn{3}{|c|}{ Cultivars } \\
\hline & & $\begin{array}{l}\text { Lleuque- } \\
\text { INIA }\end{array}$ & $\begin{array}{l}\text { Llareta- } \\
\text { INIA }\end{array}$ & $\begin{array}{c}\text { Corcolén- } \\
\text { INIA }\end{array}$ \\
\hline & & & $\%-$ & 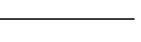 \\
\hline \multirow[t]{6}{*}{ La Platina } & 2004 & 5 & 1 & 5 \\
\hline & 2005 & 10 & 5 & 5 \\
\hline & 2006 & 1 & 0 & 5 \\
\hline & 2007 & 10 & 5 & 10 \\
\hline & 2008 & 0 & 0 & 0 \\
\hline & Mean & 5.2 & 2.2 & 5.0 \\
\hline \multirow[t]{6}{*}{ Chillán } & 2004 & 5 & 5 & 5 \\
\hline & 2005 & 20 & 20 & 20 \\
\hline & 2006 & 5 & 1 & 1 \\
\hline & 2007 & 10 & 5 & 2 \\
\hline & 2008 & 20 & 5 & 30 \\
\hline & Mean & 12 & 7.2 & 11.6 \\
\hline \multirow[t]{6}{*}{ Yungay } & 2004 & 0 & 0 & 0 \\
\hline & 2005 & 0 & 0 & 0 \\
\hline & 2006 & 20 & 20 & 20 \\
\hline & 2007 & 0 & 2 & 0 \\
\hline & 2008 & 0 & 0 & 0 \\
\hline & Mean & 4 & 4.4 & 4 \\
\hline \multirow[t]{6}{*}{ Humán } & 2004 & 0 & 0 & 0 \\
\hline & 2005 & 0 & 0 & 0 \\
\hline & 2006 & 5 & 0 & 1 \\
\hline & 2007 & 0 & 0 & 0 \\
\hline & 2008 & 5 & 0 & 0 \\
\hline & Mean & 2 & 0 & 0.2 \\
\hline
\end{tabular}

Mean values of two replicates.

From each sample, $50 \mathrm{~g}$ of grain was weighed and the grains with and without yellow berries were separated. The percentage was calculated by weight differences.
Table 8. Black tip (\%) of cv. Lleuque-INIA compared to control cvs. Llareta-INIA and Corcolén-INIA standard trials conducted in four irrigated locations from 2004 to 2008 .

\begin{tabular}{|c|c|c|c|c|}
\hline \multirow[b]{2}{*}{ Location } & \multirow[b]{2}{*}{ Year } & \multicolumn{3}{|c|}{ Cultivars } \\
\hline & & $\begin{array}{l}\text { Lleuque- } \\
\text { INIA }\end{array}$ & $\begin{array}{c}\text { Llareta- } \\
\text { INIA }\end{array}$ & $\begin{array}{c}\text { Corcolén- } \\
\text { INIA }\end{array}$ \\
\hline & & & $-\%$ & 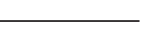 \\
\hline \multirow[t]{6}{*}{ La Platina } & 2004 & 5 & 5 & 10 \\
\hline & 2005 & 1 & 1 & 1 \\
\hline & 2006 & 0 & 0 & 1 \\
\hline & 2007 & 0 & 5 & 5 \\
\hline & 2008 & 0 & 0 & 0 \\
\hline & Mean & 1.2 & 2.2 & 3.4 \\
\hline \multirow[t]{6}{*}{ Chillán } & 2004 & 0 & 0 & 1 \\
\hline & 2005 & 1 & 5 & 1 \\
\hline & 2006 & 0 & 0 & 0 \\
\hline & 2007 & 0 & 0 & 5 \\
\hline & 2008 & 0 & 0 & 0 \\
\hline & Mean & 0.2 & 1 & 1.4 \\
\hline \multirow[t]{6}{*}{ Yungay } & 2004 & 0 & 0 & 0 \\
\hline & 2005 & 1 & 10 & 10 \\
\hline & 2006 & 0 & 10 & 5 \\
\hline & 2007 & 2 & 5 & 5 \\
\hline & 2008 & 0 & 0 & 0 \\
\hline & Mean & 0.6 & 5 & 4 \\
\hline \multirow[t]{6}{*}{ Humán } & 2004 & 1 & 1 & 1 \\
\hline & 2005 & 1 & 10 & 10 \\
\hline & 2006 & 5 & 10 & 5 \\
\hline & 2007 & 5 & 10 & 15 \\
\hline & 2008 & 0 & 0 & 0 \\
\hline & Mean & 2.4 & 6.2 & 6.2 \\
\hline
\end{tabular}

Mean values of two replicates.

From each sample $50 \mathrm{~g}$ of grain were weight and separated the grain with and without black tip. Percentage was calculated by weight differences.

\section{CONCLUSIONS}

This is a new spring durum wheat variety with a high yield potential, mean hectoliter weight values between 81 and $84 \mathrm{~kg} \mathrm{hL}^{-1}$, wet gluten percentages between 28.83 and $35.66 \%$, and mean protein values fluctuating between $10.47 \%$ and $12.3 \%$. The var. Lleuque-INIA has exhibited resistance to stripe rust, leaf rust, stem rust, and a low to moderate resistance to powdery mildew.

The sowing zone for this variety stretches from the Metropolitan Region to the Bío Bío Region between June and August in irrigated soils. 


\section{RESUMEN}

Lleuque-INIA, nueva variedad de trigo candeal de primavera de alto potencial de rendimiento para

Chile. La variedad de trigo candeal (Triticum turgidum var. durum L.) de primavera Lleuque-INIA proviene de un cruzamiento efectuado el año 1993 por el Programa Nacional de Trigo del Instituto de Investigaciones Agropecuarias INIA en el Centro Regional de Investigación La Platina, Santiago, Chile. Es un trigo con hábito de crecimiento erecto al estado de plántula, la planta adulta es de altura mediana y varía entre 80 y $90 \mathrm{~cm}$. La espiga es de tamaño medio, compacta, de color blanco, presenta barbas largas de color blanquizco en toda su extensión. El grano es de tamaño grande, de forma alargada, color amarillo dorado y vítreo. Sembrado a mediados de agosto en el Campo Experimental Santa Rosa ( $36^{\circ} 31^{\prime}$ S; 7 $71^{\circ} 54^{\prime}$ O), Chillán, la emisión de espigas ocurre entre los 89 y 91 días después de la siembra, siendo entre 6 a 8 días más tardío que 'Llareta-INIA' y ‘CorcolénINIA'. En La Platina 'Lleuque-INIA' tuvo un rendimiento medio $16,7 \%$ y $20,8 \%$ superior a la vars. Llareta-INIA y Corcolén-INIA, respectivamente; en la localidad de Chillán este rendimiento fue superior en 10,3\% y 10,1\%, respectivamente; por otro lado en Yungay, precordillera de Ñuble, el rendimiento de 'Lleuque-INIA' superó a 'Llareta-INIA' en $13,07 \%$ y a la var. Corcolén-INIA en 16,97\%. En la localidad de Humán el rendimiento de la var. Lleuque-INIA también superó en $15,23 \%$ y $24,03 \%$ a las variedades testigo Llareta-INIA y Corcolén-INIA, respectivamente. La var. Lleuque-INIA es un trigo de buen peso del hectolitro, buen contenido de gluten húmedo (\%) y con valores medios de proteína que fluctúan entre $10,47 \%$ y $12,3 \%$.

Palabras clave: trigo candeal, trigo duro, Triticum turgidum var. durum, Lleuque-INIA.

\section{LITERATURE CITED}

Peterson, R., J. Campbell, and A. Hannah. 1984. A diagrammatic scale for estimating rust intensity of leaves and stems of cereals. Canadian Journal of Research Section C-Botanical Sciences 26:496-500.

Ramírez, I., R. Cortazar, E. Hacke, D. Granger, y M. Zerene. 1997. Llareta-INIA, variedad de trigo candeal Triticum turgidum var. durum para la Zona Central de Chile. Agricultura Técnica 57:212-214.

Saari, E., and J. Prescott. 1975. A scale for appraising the foliar intensity of wheat diseases. Plant Disease Reporter 59:377-380. 